\title{
Estudantes de psicologia concluem a graduação com uma boa formação em autismo?
}

\author{
Cristiane Silvestre Paula' \\ Universidade Presbiteriana Mackenzie, São Paulo - SP - Brasil \\ José Ferreira Belisásio Filho \\ Universidade Federal de Minas Gerais, Belo Horizonte - MG - Brasil \\ Maria Cristina Triguero Veloz Teixeira \\ Universidade Presbiteriana Mackenzie, São Paulo - SP - Brasil
}

\begin{abstract}
Resumo: Considerando a importância do psicólogo na identificação/assistência a pessoas com Transtornos do Espectro Autista (TEA), o principal objetivo deste estudo foi descrever o nível de conhecimento sobre TEA de estudantes de psicologia. Estudo do tipo seccional com 85 alunos do último ano de cursos de psicologia de cinco universidades públicas e particulares de São Paulo. Os sujeitos responderam a um questionário com I I questões de múltipla escolha, sendo os principais resultados: I. bom conhecimento na amostra geral quanto aos aspectos clínico-interventivos, mas insuficiente conhecimento em epidemiologia e etiologia; desempenho discretamente superior; 2 . na temática clínico-interventiva (média 3,2 × 2,5; $\mathrm{p}=0,0 \mathrm{I}$ ) dos estudantes de universidades particulares; e 3. na temática epidemiologia (média $3,4 \times 2,3 ; p=0,0 \mathrm{I}$ ), entre alunos que haviam realizado Iniciação Científica. Esta pesquisa alerta para a necessidade de atualização permanente das propostas curriculares dos cursos de Psicologia para preparar adequadamente os futuros profissionais para adequado cuidado das pessoas com TEA.
\end{abstract}

Palavras-chave: transtorno autístico; conhecimento; estudantes de psicologia; capacitação de recursos humanos em saúde; treinamento.

\section{DID STUDENTS CONCLUDE UNDERGRADUATE DEGREE IN PSYCHOLOGY WITH A GOOD TRAINING IN AUTISM?}

\begin{abstract}
Psychologist is a key-professional in the identification/assistance of people with Autism Spectrum Disorders (ASD). Therefore, the main objective of the current study was to describe the level of knowledge on ASD of undergraduate students in Psychology. It is a cross-sectional study with 85 undergraduate students in Psychology from five public and private universities of São Paulo. Participants answered a questionnaire with II structured questions and main results were: I. good knowledge in the overall sample about clinical-intervention aspects, but insufficient knowledge on Epidemiology and etiology; performance slightly superior on; 2 . the clinical-intervention topic of students from private universities (average $3.2 \times 2.5 ; p=.01$ ); and 3 . on the Epidemiology topic of students who had concluded a scientific initiation (average $3.4 \times 2.3$; $\mathrm{p}=.0 \mathrm{I}$ ). This study points out to the need for continuous updating of curricula of Psychology undergraduate courses to prepare future professionals to properly assist people with ASD.
\end{abstract}

Keywords: autism; knowledge; undergraduate students in psychology; health human resource training; training.

${ }^{1}$ Endereço para correspondência: Cristiane Silvestre Paula, Pós-Graduação em Distúrbios do Desenvolvimento do Centro de Ciências Biológicas e da Saúde, Universidade Presbiteriana Mackenzie, Rua da Consolação, 930, Prédio 28 - São Paulo - SP - Brasil. CEP: 01302-907. E-mail: csilvestrep09@gmail.com. 
ESTUDIANTES DE PSICOLOGÍA CONCLUYEN LA LICENCIATURA CON UNA BUENA FORMACIÓN EN AUTISMO?

\begin{abstract}
Resumen: Considerando la importancia del psicólogo en la identificación/asistencia a personas con Trastornos del Espectro Autista (TEA), el principal objetivo do estudio fue describir el nivel de conocimiento sobre TEA de estudiantes de Psicología. Estudio seccional con 85 alumnos del último año de cursos de Psicología de cinco universidades públicas/particulares de São Paulo. Los sujetos respondieron a cuestionario con II preguntas de alternativas múltiples, siendo los principales resultados: I. buen conocimiento en la muestra general en relación a aspectos clínico-interventivos, pero insuficiente conocimiento en Epidemiología y etiología; desempeño discretamente superior; 2 . en el tema clínico-interventivo (media 3,2 $\times 2,5 ; p=0,0$ I) entre estudiantes de universidades particulares; y 3 . en el tema Epidemiología (media 3,4 $\times 2,3 ; p=0,0$ ), entre alumnos que habían realizado Iniciación Científica. Esta investigación alerta para la necesidad de atualización permanente de las propuestas curriculares de los cursos de Psicología para preparar adecuadamente los futuros profesionales para un adecuado cuidado de las personas con TEA.
\end{abstract}

Palabras clave: trastorno autístico; conocimiento; estudiantes de psicología; capacitación de recursos humanos en salud; capacitación.

A quinta edição do Manual Diagnóstico e Estatístico de Transtornos Mentais (DSM-V) adotou o termo Transtornos do Espectro Autista (TEA) como categoria diagnóstica para se referir ao quadro clínico caracterizado por déficits persistentes na comunicação social e na interação social, na presença de padrões restritivos e repetitivos do comportamento, interesses ou atividades (American Psychiatric Association, 2013), condensando a antiga tríade sintomatológica em dois domínios. Assim, o diagnóstico de TEA passa a abranger as antigas categorias descritas no DSM-IV: Transtorno Autista, Transtorno de Asperger, Transtorno Desintegrativo da Infância e Transtornos Globais do Desenvolvimento sem Outra Especificação.

O quadro clínico dos TEA abrange déficits nos dois domínios citados acima, sendo esses de grande variabilidade sintomatológica e de gravidade e, por isso, uma avaliação detalhada e específica deve ser feita quando há suspeita do transtorno. Como dito anteriormente, dificuldades na comunicação social precisam estar presentes para o estabelecimento do diagnóstico de TEA. Apesar da variabilidade, alguns sintomas são considerados os mais frequentes, como: 1 . isolamento ou falta de interesse em estar com outras pessoas; 2 . déficits na atenção compartilhada (principalmente na iniciação) e em brincadeiras simbólicas; 3 . diminuição ou ausência de contato visual; 4. inabilidade para estabelecer amizades e relacionamentos afetivos; 5 . dificuldade para compreender a comunicação não verbal (Bordini, Cavicchioli, Cole, Cunha, \& Machado, 2014; Zanon, Backes, \& Bosa, 2014).

Paralelamente aos problemas no campo da comunicação social, as pessoas com TEA apresentam comportamentos restritos e repetitivos que têm diversas manifestações, comumente ligadas à adesão exagerada a rotinas na presença ou não de estereotipias (motoras ou vocais). As motoras mais frequentes são: balançar para frente e para trás, chacoalhar as mãos (flapping), andar na ponta dos pés e girar objetos. Já as estereotipias vocais mais corriqueiras são: gritos inadequados, barulhos ou sons contínuos. 
Outro aspecto comum no domínio dos comportamentos restritos/repetitivos é a presença de restrição de interesses e assuntos, onde essas pessoas demonstram interesse exagerado por determinado tema geralmente bem específico, como astronomia, datas ou itinerários de ônibus (Bordini et al., 2014; Carvalho, Paula, Teixeira, Zaqueu, \& Famá D'Antino, 2013).

Dezenas de pesquisas epidemiológicas já foram concluídas, principalmente em países desenvolvidos, sendo a melhor estimativa de prevalência de TEA entre 0,6\% e 1,0\%. A partir dos estudos, sabe-se também que: 1. a distribuição de TEA por gênero se dá na ordem de uma menina para cada quatro meninos; 2. aproximadamente $60 \%$ das pessoas com TEA têm deficiência intelectual em algum nível; 3. a reincidência entre irmãos encontra-se entre 3\% e 19\%; 4. por volta de 10\% desse grupo populacional apresentam habilidades notáveis para sua idade, em geral, altamente especializadas e restritas que destoam de forma evidente de seu funcionamento global, denominadas savants (Anagnostou et al., 2014; Elsabbagh, et al., 2012; Ozonoff et al., 2011; Paula, Ribeiro, 2011).

A etiologia dos TEA ainda não está completamente estabelecida, mas há grande concordância na literatura de que sua base é neurobiológica de origem multifatorial, com um forte componente genético, combinado com a exposição do indivíduo a eventos ambientais, particularmente nos períodos pré e perinatais e que, em $80 \%$ dos casos, o TEA é detectável até os 24 meses de vida (Anagnostou et al., 2014; Paula, Ribeiro, 2011).

A assistência às pessoas com TEA tem sido prestada no Brasil tanto no sistema privado quanto no sistema público. Em relação ao sistema público de saúde brasileiro, o SUS, o atendimento a crianças e adolescentes com TEA tem migrado paulatinamente para os Centros de Atenção Psicossocial Infantojuvenil (CAPSi), sendo esses os equipamentos mais preparados para assistir casos com TEA, já que contam com equipes multidisciplinares especializadas em saúde mental. A grande maioria dos CAPSi de todo o Brasil tem pelo menos um psicólogo em sua equipe, que é responsável por uma parcela expressiva dos atendimentos (Ronchi \& Avellar, 2010). Particularmente, na cidade de São Paulo, a Secretaria Estadual de Saúde possui duas unidades especializadas no cuidado a pessoas com TEA. O Centro de Referência em Transtornos do Espectro Autista Philippe Pinel foi inaugurado em 2010 para atender crianças e adolescentes com até 18 anos de idade. A Unidade de Referência em Transtornos do Espectro Autista Dr. Marcos T. Mercadante da Santa Casa de São Paulo foi inaugurada em 2013 e atende crianças, adolescentes e adultos com TEA. Ambos oferecem atendimento especializado com equipe multidisciplinar.

Ainda no SUS, as Unidades Básicas de Saúde (UBS) e o Programa Estratégia de Saúde da Família (ESF), equipamentos da atenção primária/básica, têm papel complementar aos cuidados prestados a crianças/jovens com TEA, pois são essenciais na identificação dos casos e, em certo nível, responsáveis por sua assistência integral. A equipe mínima que compoe o ESF é formada por um médico da família, um auxiliar de 
enfermagem e seis agentes comunitários (Paula, Lauridsen-Ribeiro, Wissow, Bordin, \& Evans-Lacko, 2012). Entretanto, existem equipes mais extensas incluindo em algumas unidades um ou mais psicólogos. Ao lado do SUS, estão as organizações de familiares de pessoas com TEA e outras entidades, conveniadas ou independentes do SUS, além de serviços estabelecidos em instiuições acadêmicas que têm um papel complementar de alta relevância, principalmente nos grandes centros urbanos. Infelizmente, não podemos apresentar informações confiáveis sobre a assistência prestada aos adultos com TEA no Brasil, pois dados nesse campo são praticamente inexsistentes.

Como não existe um marcador biológico determinante, e sabendo-se que o TEA constitui um quadro complexo, faz-se necessária a presença de uma equipe multidisciplinar experiente tanto para identificação dos casos quanto para sua assistência. Na equipe multiprofissional, o médico é o profissional responsável por realizar o diagnóstico, geralmente um psiquiatra infantil, pediatra ou neurologista. Além dele, devem compor a equipe psicólogos, fonoaudiólogos e outros profissionais da saúde e educação, dependendo da necessidade de cada caso (Anagnostou et al., 2014; Bordini et al., 2014)

O psicólogo tem um papel-chave na equipe multiprofissional, e uma boa formação em desenvolvimento típico e atípico é essencial para a boa prática de sua profissão (Bosa, 2002; Paula, Zaqueu, Vicente, Lowenhal, \& Miranda, 2011). De forma geral, em todos os serviços de saúde descritos acima, o psicólogo é um dos profissionais da equipe mais indicados para a avaliação clínico-comportamental dos TEA. Por isso, é esperado que ele esteja capacitado para escutar as queixas dos pais relacionadas aos TEA, a fim de reconhecer os sinais e sintomas característicos e diferenciais do transtorno contribuindo para a emissão correta de um diagnóstico. Nesse sentido, é desejável que sua formação, desde a graduação, inclua disciplinas e/ou conteúdos curriculares que abranjam os TEA de maneira aprofundada (Samms-Vaughan, 2014; Trembath \& Vivanti, 2014).

Além da avaliação de crianças com TEA, o psicólogo desempenha um papel central na assistência direta à criança, assim como na orientação e suporte aos pais. Não existe uma abordagem única e determinante para o tratamento de todas as pessoas com TEA, já que as intervenções são complementares e devem ser realizadas concomitantemente, levando em conta as necessidades específicas de cada criança. Por outro lado, existem intervenções com comprovada evidência de eficácia (Anagnostou et al., 2014) e, portanto, devem ser, em princípio, oferecidas para essa população, podendo ser classificadas em: 1. intervenção precoce estruturada; 2 . intervenção para minimizar problemas comportamentais; e 3. intervenção medicamentosa.

1. As intervenções precoces estruturadas têm sido as mais estudadas e são as mais promissoras no campo dos TEA. Há décadas, diversos autores vêm trabalhando no tema, sendo o pioneiro nesse campo o psicólogo Ole Ivar Lovaas que com uma vasta experiência na Análise Aplicada do Comportamento (ABA), na década de 1970, demonstrou que o comportamento de crianças com TEA pode ser modificado utilizando essa técnica. A intervenção comportamental precoce destinada a crianças com TEA 
denomina-se Early Intensive Behavioral Intervention (EIBI) ou Intervenção Comportamental Precoce Intensiva. A EIBI tem como objetivo a redução de comportamentos não adaptativos por meio da extinção e quebra do padrão básico estabelecido, tendo como principal elemento o reforço positivo de comportamentos desejados. Ela abarca diversas fases, incluindo: 1. redução de comportamentos indesejados; 2 estimulação de comportamentos desejados por meio, por exemplo, de imitação; 3. integração dos familiares na intervenção visando futuramente que participem como coterapeutas; e 4. preparo para inclusão escolar. Já foram publicadas três meta-análises compilando resultados de pesquisas sobre ABA e EIBI (Eldevik, Hastings, Hughes, Jahr, Eikeseth, \& Cross, 2009; Reichow, 2012; Virués-Ortega, 2010), o que nos permite fazer uma avaliação crítica dos resultados. Nessas meta-análises estão incluídos 29 artigos nos quais foram analisadas 1.008 crianças com TEA, e os principais resultados foram que: 1. intervenções mais longas e intensivas levam a melhores resultados ( $\geq 27$ horas/semana e superiores há 24 meses); 2. a maioria dos estudos identificou mudanças estatisticamente significantes na pontuação média de QI e nas habilidades comunicativas; 3. nem todos os 29 estudos incluíram avaliações de funcionamento adaptativo e de linguagem, mas, entre aqueles que o fizeram, a maioria obteve resultados estatisticamente positivos; 4. a inclusão das crianças em salas regulares se deu com sucesso em metade das crianças, sendo $50 \%$ sem necessidade de suporte pedagógico especializado. Esses resultados são bem positivos, mas algumas limitações metodológicas dos estudos precisam ser relatadas: 1. a média de participantes em cada estudo foi pequena (38,8 crianças/estudo); 2 . em vários estudos não há um grupo controle; e 3. as intervenções com as quais a EIBI foi comparada eram muito variadas e, em geral, compostas por diversos tratamentos, limitando as conclusões.

Grande parte das crianças com TEA apresentam problemas comportamentais que, se não forem tratados, tendem a se agravar e levar a outras complicações, tanto para a criança como para os seus familiares. Comportamentos agressivos, birras e estereotipias estão entre aqueles passíveis de intervenção. Vale ressaltar que geralmente essas intervenções devem ser planejadas com o objetivo de trabalhar comportamentos específicos eleitos como os mais prejudiciais sem uma proposta de abarcar o desenvolvimento infantil de forma mais global (Agency for Healthcare Research and Quality, 2014; Horner, Carr, Strain, Todd, \& Reed, 2002). Um estudo de revisão revela que essas intervenções comportamentais específicas podem levar à redução de mais de $80 \%$ desses comportamentos. Além disso, na maioria dos casos, os resultados permanecem estáveis nos meses seguintes ao final da intervenção. Todavia, a generalização desses resultados promissores para outros ambientes não estruturados ainda não tem evidência de eficácia comprovada (Horner et al., 2002).

Além das terapias coordenadas por psicólogos ou outros profissionais da saúde, em alguns casos, o tratamento medicamentoso é indicado para pessoas com TEA. A responsabilidade por esse tipo de tratamento é do médico da equipe e deve sempre ser elaborado de acordo com um plano individualizado. Ainda não existem psicofárma- 
cos, com sólida evidência de eficácia, destinados ao tratamento dos sintomas nucleares dos TEA, já que dificuldades na interação social e na comunicação têm se mostrado resistentes a terapias farmacológicas. Por outro lado, certos sintomas frequentes nesse quadro clínico, como aqueles relacionados à irritabilidade, à hiperatividade e desatenção, à autoagressão, à insônia, a comportamentos repetitivos, têm obtido resultados positivos decorrentes de tratamento medicamentoso (Anagnostou et al., 2014; Gibbs, 2010). É importante ressaltar que o tratamento medicamentoso deve focar nos sintomas específicos identificados em cada indivíduo, ou seja, o profissional deve elaborar uma terapêutica conjuntamente com a família e baseada nos sintomas mais disfuncionais. Finalmente, os efeitos colaterais devem ser observados e levados em consideração na continuidade ou suspensão da medicação. Além disso, o tratamento medicamentoso deve ser combinado com outros tipos de intervenção, como psicoterapias, terapias fonoaudiológicas, pedagógicas, entre outras.

Em síntese, é possível afirmar que as intervenções mais efetivas são aquelas iniciadas precocemente, de caráter intensivo, individualizado, padronizado, de longa duração e conduzido por uma equipe multiprofissional.

Considerando a importância do psicólogo na identificação e assistência a pessoas com TEA, espera-se que esse profissional tenha uma boa formação em todos os aspectos abordados anteriormente para que seja capaz de assistir adequadamente crianças e adolescentes com TEA. Assim, os objetivos do presente estudo foram descrever o nível de conhecimento sobre TEA de estudantes de graduação em psicologia; e testar diferenças no tipo de conhecimento em função: 1. da origem dos cursos (universidades públicas ou particulares); e 2. entre universitários que haviam realizado ou não Iniciação Científica.

\section{Método}

Adotou-se um delineamento de tipo seccional com uma amostra de conveniência de 85 alunos de cursos de Psicologia dos $9^{\circ}$ e $10^{\circ}$ semestres de quatro universidades particulares e uma universidade pública, todas da cidade de São Paulo. Os estudantes foram recrutados por meio de convites em sala de aula e nas redes sociais. Do total de participantes, 76,5\% estudavam em universidades particulares, 77,6\% eram mulheres e tinham em média 26 anos de idade (DP $\pm 9,3$ ). O projeto foi aprovado pela Comissão de Ética em Pesquisa da Universidade Presbiteriana Mackenzie (Protocolo CIEP n. P025/11/12).

\section{Instrumentos e procedimentos}

O primeiro passo da pesquisa foi a elaboração de um questionário objetivo composto por 11 questões de múltipla escolha, abarcando as quatro grandes áreas apresentadas na introdução deste artigo e consideradas essenciais para a formação em TEA de um psicólogo: 1. Epidemiologia (quatro questões, incluindo prevalência, inci- 
dência por gênero, taxas de Deficiência Intelectual entre pessoas com TEA e reincidência do transtorno entre irmãos com TEA); 2. Etiologia (uma questão); 3. Perfil Clínico e Intervenção (quatro questões, sendo duas sobre aspectos clínicos dos TEA, uma sobre abordagem psicoterápicas baseadas em evidência e uma sobre sinais/sintomas que são minimizados ante o tratamento medicamentoso); 4. Áreas de avaliação em crianças com suspeita de TEA (uma questão); e 5. Serviços Públicos de Saúde (uma questão sobre qual o equipamento do SUS mais preparado para atender crianças/jovens com TEA). Além dessas 11 questões, havia uma questão sobre a impressão subjetiva dos estudantes sobre seu nível de conhecimento em TEA.

Os questionários foram distribuídos nas salas de aulas dos estudantes de psicologia e cada um o respondia de forma autoaplicável. Antes disso, todos os participantes foram plenamente esclarecidos sobre os objetivos do estudo e sobre o tipo de instrumento que responderiam. Os que concordaram em participar assinaram o termo de consentimento livre e esclarecido e, posteriormente, passaram a responder ao questionário. Foi garantido o sigilo das informações prestadas pelos participantes. As coletas aconteceram em 2013, em salas de aula das universidades que os participantes frequentavam. Durante a aplicação, a equipe de pesquisa permaneceu nas salas para acompanhar o preenchimento do questionário e sanar possíveis dúvidas.

\section{Procedimentos de análise de dados}

Os dados foram organizados num banco no programa SPSS versão 19.0. Inicialmente, foi feita uma análise descritiva dos dados visando caracterizar o perfil de conhecimento em TEA de todos os sujeitos da pesquisa. Posteriormente, foram realizadas análises bivariadas para testar diferenças entre o nível de conhecimento em TEA segundo o tipo de universidade que o sujeito estudava (pública ou particular), assim como entre aqueles que haviam ou não realizado Iniciação Científica. Para tanto, foram aplicados testes de diferença de média (Teste $\mathrm{T}$ ) nas variáveis contínuas com distribuição normal e o teste não paramétrico de Qui-Quadrado nas variáveis categóricas, sendo calculada a significância de acordo com o Teste de Pearson ou Teste Exato de Fisher, quando aplicável. Adotou-se o nível de probabilidade de $95 \%(p \leq 0,05)$ para a rejeição das hipóteses de nulidade.

\section{Resultados}

Inicialmente, apresentaremos os índices de acerto de todos os participantes da pesquisa, segundo cada item do questionário (Tabela 1) e, em seguida, esse mesmo índice segundo duas temáticas (Tabela 2). Posteriormente, serão apresentadas as análises inferenciais buscando diferenças entre estudantes de universidades públicas e particulares, e entre estudantes que haviam ou não realizado Iniciação Científica (IC) na área da saúde mental da infância e adolescência durante o curso de graduação (tabelas 3 e 4). A maioria desses universitários $(62,4 \%)$ avaliou seu conhecimento em TEA como 
insuficiente, 27,1\% afirmaram ter um conhecimento regular e, apenas, 5,9\% consideraram seu nível de conhecimento bom.

$\mathrm{Na}$ amostra total do estudo, observam-se índices de acerto bem variados considerando cada uma das 11 perguntas do questionário. De forma geral, nota-se que os sujeitos tendem a errar menos questões na área clínica dos TEA (sintomatologia e suas abordagens terapêuticas e medicamentosas) e relacionada ao sistema público de saúde (78,8\% de acerto). Por outro lado, os alunos erram mais as perguntas especializadas no campo dos TEA, ou seja, aquelas que dependem de uma formação técnica como é o caso da epidemiologia, área na qual se identificaram os piores índices de acerto: 14,1\% sobre a taxa de prevalência, $11,8 \%$ sobre os índices de recorrência entre irmãos e $8,2 \%$ sobre a incidência de deficiência intelectual nas pessoas com TEA. Destaca-se também o baixo índice de acerto sobre a etiologia dos TEA $(9,4 \%)$ (Tabela 1$)$.

Outro dado que também chama a atenção nesta pesquisa é que em quatro das 11 perguntas do questionário, uma parcela expressiva dos participantes (entre 34,0\% e 44,3\%) respondeu espontaneamente "não sei", indicando que eles não tinham nenhuma ideia sobre o assunto em pauta.

Tabela I. Distribuição de acertos por questão $(\mathbf{N}=85)$

\begin{tabular}{ll} 
Questão & $\%$ de acerto \\
\hline Principal unidade de atendimento na rede de pública de saúde & 78,8 \\
\hline Abordagens terapêuticas baseadas em evidência & 76,5 \\
\hline Sintomas minimizados pela medicação & 71,8 \\
\hline Perfil clínico de indivíduos com Asperger & 64,7 \\
\hline O que significam as habilidades Savant & 52,9 \\
\hline Domínios que devem ser avaliados nos TEA & 35,3 \\
\hline Incidência por sexo & 35,3 \\
\hline Prevalência de TEA & 14,1 \\
\hline Recorrência entre irmãos & 11,8 \\
\hline Etiologia dos TEA & 9,4 \\
\hline Incidência de deficiência intelectual entre pessoas com TEA & 8,2 \\
\hline
\end{tabular}

Fonte: Elaborada pelos autores.

Considerando as áreas de maior e menor conhecimento identificadas nesta amostra, assim como sua relevância para a prática do futuro psicólogo, decidiu-se por criar dois blocos temáticos: "Perfil clínico/Intervenção" e "Epidemiologia". No primeiro bloco, foram incluídas quatro questões: sobre os sintomas clínicos das pessoas com 
Asperger e o significado das habilidades Savants, assim como sobre o tratamento que deve ser prestado aos TEA, de acordo com evidências científicas (abordagens psicológicas e medicamentosas). No segundo bloco, foram incluídas outras quatro questões relativas ao perfil epidemiológico dos indivíduos com TEA, sendo elas: taxa de prevalência, incidência por sexo, recorrência entre irmãos e taxa de deficiência intelectual nos TEA. Quando analisamos os dados segundo essas duas temáticas, notamos um bom conhecimento dos universitários quanto aos aspectos clínicos e de intervenção, já que $60 \%$ dos sujeitos acertaram entre $75 \%$ e $100 \%$ das perguntas, mas um insuficiente conhecimento em epidemiologia, em que quase $90 \%$ acertaram entre zero e $25 \%$ dos itens e nenhum acertou as quatro questões (Tabela 2).

Tabela 2. Distribuição de acertos por tema $(\mathbf{N}=\mathbf{8 5})$

\begin{tabular}{llllll} 
& $0 \%$ & $25 \%$ & $50 \%$ & $75 \%$ & $100 \%$ \\
Temas & $\mathrm{N}(\%)$ & $\mathrm{N}(\%)$ & $\mathrm{N}(\%)$ & $\mathrm{N}(\%)$ & $\mathrm{N}(\%)$ \\
\hline Sintomatologia & $\mathrm{I}(1,2)$ & $\mathrm{II}(12,9)$ & $22(25,9)$ & $33(38,8)$ & $\mathrm{I}(2 \mathrm{I}, 2)$ \\
\hline Epidemiologia & $37(43,5)$ & $39(45,9)$ & $8(9,4)$ & $\mathrm{I}(1,2)$ & ------ \\
\hline
\end{tabular}

Fonte: Elaborada pelos autores.

A partir da Tabela 3, é possível afirmar que praticamente não há diferenças no nível de conhecimento em TEA entre os estudantes de diferentes tipos de universidades. Em apenas uma das 11 perguntas do questionário observa-se que aqueles que frequentavam a universidade pública apresentam quatro vezes mais chances de acertarem a pergunta sobre "o perfil clínico das pessoas com Asperger" que os estudantes de escolas particulares ( $p=0,03$; IC95\%: 1,07-15,12).

Apenas 30 dos 85 sujeitos da pesquisa (35,2\%) haviam concluído IC, porém, somente três deles no campo da saúde mental infantil e/ou autismo. Verificamos que não existe nenhuma diferença no nível de conhecimento em TEA dos estudantes que haviam concluído um trabalho de IC na área desta pesquisa em comparação com seus colegas (Tabela 3), tampouco se verificou diferença entre o fato de terem realizado IC em qualquer campo do conhecimento em relação aos colegas que não haviam realizado IC (dado não apresentado). 
Tabela 3. Comparação entre o nível de conhecimento em TEA de estudantes de faculdades públicas e particulares; e entre ter realizado ou não Iniciação Científica (IC), segundo análise bivariada $(\mathbf{N}=\mathbf{8 5})$

\begin{tabular}{|c|c|c|c|c|c|c|c|c|}
\hline \multirow[b]{2}{*}{ Itens do questionário sobre TEA } & \multicolumn{4}{|c|}{ Universidade } & \multicolumn{4}{|c|}{ Realizou IC* } \\
\hline & $\begin{array}{l}\text { Pública } \\
\text { N (\%) }\end{array}$ & $\begin{array}{l}\text { Particular } \\
\text { N (\%) }\end{array}$ & $\begin{array}{c}\text { OR } \\
\text { (IC 95\%) }\end{array}$ & $\mathrm{p}$ & $\begin{array}{c}\text { Sim } \\
\mathrm{N}(\%)\end{array}$ & $\begin{array}{c}\text { Não } \\
\text { N (\%) }\end{array}$ & $\begin{array}{c}\text { OR } \\
\text { (IC 95\%) }\end{array}$ & $\mathrm{p}$ \\
\hline \multicolumn{9}{|l|}{ Unidade de atendimento no SUS } \\
\hline Acertou & $16(80,0)$ & $51(78,5)$ & 1,10 & $\mathrm{I}, 00$ & $3(100,0)$ & $64(78,3)$ & 0,96 & 1,00 \\
\hline Errou & $4(20,0)$ & $14(2 \mid, 5)$ & $(0,32-3,8 I)$ & & $0(0,0)$ & $18(22,0)$ & $(0,9 I-I, 0 I)$ & \\
\hline \multicolumn{9}{|l|}{ Abordagens terapêuticas } \\
\hline \multicolumn{9}{|l|}{ baseadas em evidência } \\
\hline Acertou & $18(90,0)$ & $47(72,3)$ & 3,45 & 0,14 & $3(100,0)$ & $62(75,6)$ & 0,95 & $\mathrm{I}, 00$ \\
\hline Errou & $2(10,0)$ & I8 $(27,7)$ & $(0,73-16,38)$ & & $0(0,0)$ & $20(24,4)$ & $(0,90-I, 0 I)$ & \\
\hline \multicolumn{9}{|l|}{ Medicação } \\
\hline Acertou & $17(85,0)$ & $44(67,7)$ & 2,71 & 0,13 & $2(66,7)$ & $59(72,0)$ & 0,78 & 1,00 \\
\hline Errou & $3(15,0)$ & $21(32,3)$ & $(0,7 \mid-10,26)$ & & I $(33,3)$ & $23(28,0)$ & $(0,69-9,02)$ & \\
\hline \multicolumn{9}{|l|}{ Perfil clínico de Asperger } \\
\hline Acertou & $17(85,0)$ & $38(58,5)$ & 4,03 & 0,03 & $2(66,7)$ & $7(53,8)$ & 1,74 & 1,00 \\
\hline Errou & $3(15,0)$ & $27(4 \mid, 5)$ & $(1,07-15,12)$ & & I $(33,3)$ & $6(46,2)$ & $(0,12-23,94)$ & \\
\hline \multicolumn{9}{|l|}{ Habilidades Savant } \\
\hline Acertou & $12(60,0)$ & $33(50,8)$ & 1,46 & 0,47 & $2(66,7)$ & $43(52,4)$ & $\mathrm{I}, 8 \mathrm{I}$ & 1,00 \\
\hline Errou & $8(40,0)$ & $32(49,2)$ & $(0,53-4,03)$ & & I $(33,3)$ & $39(47,6)$ & $(0,16-20,80)$ & \\
\hline \multicolumn{9}{|l|}{ Domínios de avaliação } \\
\hline Acertou & $9(45,0)$ & $21(32,3)$ & $|, 7|$ & 0,30 & I $(33,3)$ & $29(35,4)$ & 0,92 & $\mathrm{I}, 00$ \\
\hline Errou & II $(55,0)$ & $44(67,7)$ & $(0,62-4,77)$ & & $2(66,7)$ & $53(64,6)$ & $(0,08-|0,5|)$ & \\
\hline \multicolumn{9}{|l|}{ Incidência por sexo } \\
\hline Acertou & $6(30,0)$ & $24(36,9)$ & 0,73 & 0,57 & $3(100,0)$ & $27(32,9)$ & 0,90 & 0,41 \\
\hline Errou & $14(70,0)$ & $4 I(63, I)$ & $(0,25-2,16)$ & & $0(0,0)$ & $55(67, I)$ & $(0,80-I, 04)$ & \\
\hline \multicolumn{9}{|l|}{ Prevalência } \\
\hline Acertou & I $(5,0)$ & II $(16,9)$ & 0,26 & 0,28 & I $(33,3)$ & II $(13,4)$ & 3,23 & 0,33 \\
\hline Errou & $19(95,0)$ & $54(83, I)$ & $(0,03-2,14)$ & & $2(66,7)$ & $7 \mid(86,6)$ & $(0,27-38,66)$ & \\
\hline \multicolumn{9}{|l|}{ Recorrência entre irmãos } \\
\hline Acertou & $2(10,0)$ & $8(12,3)$ & 0,79 & $\mathrm{I}, 00$ & $0(0,0)$ & $10(12,2)$ & 1,04 & 1,00 \\
\hline Errou & $18(90,0)$ & $57(87,7)$ & $(0,15-4,07)$ & & $3(100,0)$ & $72(87,8)$ & $(0,99-I, 09)$ & \\
\hline \multicolumn{9}{|l|}{ Etiologia } \\
\hline Acertou & $4(20,0)$ & $4(6,2)$ & 3,81 & 0,08 & $0(0,0)$ & $8(9,8)$ & 1,04 & $\mathrm{I}, 00$ \\
\hline Errou & $16(80,0)$ & $47(93,8)$ & $(0,86-16,94)$ & & $3(100,0)$ & $74(90,2)$ & $(0,99-I, 09)$ & \\
\hline \multicolumn{9}{|l|}{ Incidência de DI } \\
\hline Acertou & $2(10,0)$ & $5(7,7)$ & 1,33 & 0,67 & I $(33,3)$ & $6(7,3)$ & 6,33 & 0,23 \\
\hline Errou & $18(90,0)$ & $60(92,3)$ & $(0,24-7,46)$ & & $2(66,7)$ & $76(92,7)$ & $(0,50-80-32)$ & \\
\hline
\end{tabular}

Fonte: Elaborada pelos autores. 
Por outro lado, quando analisamos esses mesmos grupos, segundo as temáticas "Perfil clínico/Intervenção" e "Epidemiologia", algumas diferenças tornam-se estatisticamente significantes. Na Tabela 4, verifica-se que os graduandos de universidades particulares acertaram mais as questões de âmbito clinico/interventivo (média $3,2 \times 2,5 ; p=0,01$ ) que os graduandos de universidades públicas; do mesmo modo, constata-se que os alunos que haviam realizado IC na área da saúde mental infantil e/ou autismo acertaram mais as questões relativas à epidemiologia (média $1,0 \times 1,4 ; p=0,01$ ) que seus colegas.

Tabela 4. Comparação entre o nível de conhecimento em TEA de estudantes de faculdades públicas e particulares e entre ter realizado ou não Iniciação Científica (IC) segundo teste de diferença de médias $(\mathbf{N}=85)$

\begin{tabular}{|c|c|c|c|c|c|c|}
\hline & \multicolumn{3}{|c|}{ Temática Perfil Clínica/intervenção } & \multicolumn{3}{|c|}{ Temática epidemiologia } \\
\hline & $\begin{array}{l}\text { Média de } \\
\text { acerto }\end{array}$ & Valor de $\mathrm{p}$ & IC $95 \%$ & $\begin{array}{l}\text { Média de } \\
\text { acerto }\end{array}$ & Valor de $\mathrm{p}$ & IC 95\% \\
\hline \multicolumn{7}{|c|}{ Tipo de universidade } \\
\hline Pública & 3,2 & 0,01 & 0,$21 ; 1,20$ & 0,5 & 0,18 & 0,$59 ; 0,11$ \\
\hline Particular & 2,5 & & & 0,7 & & \\
\hline \multicolumn{7}{|c|}{ IC } \\
\hline Fez & 1,0 & 0,55 & $-1,52 ; 0,81$ & 2,3 & 0,01 & $-I, 8 I ;-0,24$ \\
\hline Não fez & $\mathrm{I}, 4$ & & & 3,4 & & \\
\hline
\end{tabular}

Fonte: Elaborada pelos autores.

\section{Discussão}

Pesquisas científicas são promissoras ao indicar que a identificação precoce, seguida de uma assistência de qualidade, prediz um melhor prognóstico de crianças com TEA (Werner, Dawson, Munson, \& Osterling, 2005) e, consequentemente, leva à redução de custos financeiros e sociais para as famílias e para os sistemas públicos de educação e saúde. Nesse sentido, os psicólogos que atuam no SUS são equipamentos fundamentais na identificação precoce de casos com o transtorno e na implantação de intervenções adequadas.

Existem Diretrizes Curriculares Nacionais para os cursos de graduação em Psicologia, a serem observadas pelas instituições de ensino superior do país. Uma delas direciona-se a dotar o profissional de conhecimentos adequados para o exercício de um conjunto de competências e habilidades gerais. Especificamente na atenção à saúde, espera-se que esses profissionais estejam aptos a desenvolver ações de prevenção, promoção, proteção e reabilitação da saúde psicológica e psicossocial, em nível tanto individual quanto coletivo, bem como a realizar seus serviços dentro dos mais altos padrões de qualidade e dos princípios da ética/bioética (Ministério da Educação, Con- 
selho Nacional Superior, 2011). Mas cabe aos cursos de graduação formatar as disciplinas do núcleo comum de formação seguindo um dado conteúdo programático, preferencialmente com tratamentos baseados em evidências científicas, nas diferentes áreas de conhecimentos psicológicos. Não foi possível analisar o conteúdo programático relativo às disciplinas de saúde dos cursos de psicologia dos sujeitos desta pesquisa, portanto, desconhecemos a quantidade e a qualidade de conteúdo sobre TEA oferecidas nesses cursos. Essa é a primeira limitação da pesquisa a ser relatada.

O percentual de acertos verificado nas diferentes questões do instrumento mostra uma série de conhecimentos essenciais para a formação de um psicólogo clínico que poderá ter exigências profissionais ligadas à detecção de sinais de TEA, à identificação de condições associadas ao transtorno, seus aspectos conceituais e de intervenção, tanto na rede pública quanto na rede privada. Entretanto, esse conhecimento se mostrou irregular na amostra. Os participantes estão relativamente bem informados quanto aos locais de atendimento na rede pública, sobre intervenções eficazes e sobre a eficácia no uso de medicação. Já os conhecimentos gerais sobre o quadro clínico dos TEA, etiologia e dados epidemiológicos foram regulares/baixos.

Tomando como exemplo um dos dados da Tabela 1, verifica-se que apenas 8,2\% da amostra souberam identificar o grau de associação do TEA com a deficiência intelectual. Isso pode comprometer o futuro desempenho profissional em diferentes alvos de trabalho do psicólogo; por exemplo, avaliação neuropsicológica, avaliação comportamental e avaliação de funcionamento adaptativo. Além disso, apenas 35,3\% dos participantes sabiam quais os domínios que precisam ser avaliados ante uma suspeita de TEA, campo bem específico da psicologia. Ao mesmo tempo, apesar de o grau de conhecimentos sobre aspectos ligados à intervenção ter sido bem superior $(76,5 \%$ de acerto no item "Abordagens terapêuticas baseadas em evidência" e 71,8\% na questão "Sintomas minimizados pela medicação"), era esperado um desempenho ainda meIhor em tópicos centrais do campo de atuação do psicólogo clínico, já que os sujeitos da pesquisa em breve estarão no mercado de trabalho. Assim, nota-se que alguns aspectos essenciais na formação dos psicólogos parecem estar deficitários. Como já relatado anteriormente, o psicólogo tem papel-chave na equipe multiprofissional que atende pessoas com TEA, portanto, deveria estar bem preparado para responder a todos os itens do questionário, mostrando-se capaz de identificar desvios nos marcos do desenvolvimento e, consequentemente, podendo propor as terapêuticas mais adequadas para cada caso (Bosa, 2002).

A Iniciação Científica, como o próprio nome diz, costuma ser o primeiro passo para a formação mais especializada da carreira científico-acadêmica. Uma parcela crescente dos universitários brasileiros tem tido essa oportunidade e, na amostra estudada, esse índice foi de 35,2\% ao longo da graduação. Todavia, os temas de IC relatados eram muito diversos e pouco relacionados com os TEA, por isso, não nos supreendeu que os índices de acerto tenham sido semelhantes entre os que haviam ou não realizado IC. Por outro lado, mesmo com um número tão baixo de estudantes que 
havia concluído um projeto de IC na área da saúde mental infantil e/ou autismo (três alunos), foi possivel identificar um melhor conhecimento desses no bloco temático da Epidemologia dos TEA. Isso indica que a IC traz realmente um benefício na formação dos universitários e que deve ser estimulada logo nos primeiros anos da graduação.

A presente pesquisa também revelou que o nível de conhecimentos em TEA foi bastante semelhante entre o grupo oriundo de universidades particulares e públicas, com exceção do bloco temático da Epidemiologia, onde os alunos de universidade pública apresentaram um conhecimento superior ao de seus colegas. Portanto, parece que mais importante do que o tipo de universidade que o aluno frequenta, a experiência de realizar uma IC e o conteúdo programáticos do núcleo comum dos currículos das universidades devem ter um impacto mais relevante em seu aprendizado. Todavia, sendo este o primeiro estudo brasileiro nessa temática, novas pesquisas devem ser realizados para confirmar essa hipótese.

Segundo nossa revisão da literatura, nenhum estudo anterior foi concluído analisando dados semelhantes aos da presente pesquisa. Nesta revisão, foi identificado um único estudo similar que investigou conhecimentos sobre etiologia, sintomatologia/diagnóstico e tratamento para autismo entre estudantes de medicina de uma universidade do Rio Grande do Sul (Muller, 2012). Os resultados daquela pesquisa apontaram conhecimentos deficitários dos participantes, independentemente de estarem cursando o 1o ou o 6a ano do curso, revelando uma limitação na formação médica. Os resultados de nosso estudo apontam na mesma direção. Psicólogos adequadamente capacitados precisam ter conhecimentos básicos sobre TEA, dentre eles, taxas de prevalência, recorrência familiar do transtorno e condições psiquiátricas associadas, por exemplo a DI. Apesar disso, a maioria dos participantes do estudo não soube responder a essas perguntas, o que pode comprometer suas ações no campo clínico, assim como na gestão de serviços públicos (Soltau, Biedermann, Hennicke, \& Fydrich, 2015). São competências requeridas do psicólogo e que, por essa razão, deveriam fazer parte do cotidiano de aulas e da busca de informação bibliográfica sobre o tema nos diversos indexadores, periódicos, livros e outras fontes especializadas.

Apesar de este artigo contribuir com dados inéditos, ele apresenta algumas limitações, como ser baseado em amostra de conveniência com tamanho reduzido, o que impede a generalização dos dados; e ter utilizado questionário desenvolvido pela equipe de pesquisa sem antes ter testado suas evidências de validade. Portanto, recomendam-se estudos futuros com amostras aleatórias maiores e representativas de estudantes de psicologia de diferentes regiões do país que ajudem a apreender especificidades em relação à formação em psicologia nesse tipo de transtorno. Concomitantemente, podem ser comparados esses dados com os conteúdos curriculares das disciplinas dedicadas a desenvolvimento humano nos cursos que permitam identificar com maior precisão as lacunas nas propostas dos cursos. 
Apesar dessas recomendações, os dados obtidos permitem alertar para a necessidade de atualização permanente das propostas curriculares dos projetos pedagógicos dos cursos de psicologia, em consonância com evidências científicas para abordagem dos TEA. Somente conteúdos curriculares aprofundados e baseados em evidências científicas garantirão um núcleo básico de competências e uma prática profissional de alto nível.

\section{Referências}

Agency for Healthcare Research and Quality. (2014). Therapies for children with autism spectrum disorder: behavioral interventions update. Rockville: Agency for Healthcare Research and Quality.

American Psychiatric Association (2013). Diagnostic and statistical manual of mental disorders. Washington: American Psychiatric Association.

Anagnostou, E., Zwaigenbaum, L., Szatmari, P., Fombonne, E., Fernandez, B. A., Woodbury-Smith, M., \& Scherer, S. W. (2014). Autism spectrum disorder: advances in evidence-based practice. Canadian Medical Association Journal, 186(7), 509-519.

Bordini, D., Cavicchioli, D. A. N., Cole, C. G., Cunha, G. R., \& Machado, F. S. N., (2014). Entendendo o autismo: uma visão atualizada da clínica ao tratamento. São Paulo: Conectfarma Publicações Científicas Ltda.

Bosa, C. A. (2002). Autismo: intervenções psicoeducacionais. Revista Brasileira de Psiquiatria, 28(Supl. I), 47-53.

Carvalho, F. A., Paula, C. S., Teixeira, M. C. T., Zaqueu, L. da C. C., \& Famá D'Antino, M. E. (2013). Screening of early signs of Autism Spectrum Disorder in children of a day care center from the city of São Paulo. 15(2), 144-154. Recuperado em 15 maio, 2015, de http://search.ebscohost.com/login.aspx?direct=true \&db=a9h\& AN=94785593\&lang=es\&site=ehost-live.

Eldevik, S., Hastings, R. P., Hughes, J. C., Jahr, E., Eikeseth, S., \& Cross, S. (2009). Meta-analysis of early intensive behavioral intervention for children with autism. Journal of Clinical Child \& Adolescent Psychology, 38(3), 439-450.

Elsabbagh, M., Divan, G., Koh, Y. J., Kim, Y. S., Kauchali, S., Marcín, C., \& Fombonne, E. (2012). Global Prevalence of Autism and Other Pervasive Developmental Disorders. Autism Research, 5(3), 160-179.

Gibbs, T. T. (2010). The Neurochemical Basis of Autism. In G. J. Blatt. (Ed.). The neurochemical basis of autism: from molecules to minicolumns. Boston: Springer US. Recuperado em 15 maio, 2015, de http://link.springer.com/10.1007/978$-1-4419-1272-5$. 
Horner, R. H., Carr, E. G., Strain, P. S., Todd, A. W., \& Reed, H. K. (2002). Problem behavior interventions for young children with autism: a research synthesis. Journal of Autism and Developmental Disorders, 32(5), 423-46. Recuperado em 15 maio, 2015, de http://www.ncbi.nlm.nih.gov/pubmed/12463518.

Ministério da Educação, Conselho Nacional Superior, C. de E. S. (2011). Resolução CNE/CES n. 5/2011. Diário Oficial da União, Brasília, 16 de março de 2011 - Seção 1. Recuperado em 15 maio, 2015, de http://portal.mec.gov.br/index.php?option= com_content\&id=12991: diretrizes-curricularescursos-de-graduacao.

Muller, C. (2012). Conhecimento acerca do autismo em uma Universidade do Rio Grande do Sul. Dissertação de mestrado, Programa de Pós-Graduação em Saúde da Criança e do Adolescente, Universidade do Rio Grande do Sul, RS, Brasil.

Ozonoff, S., Young, G. S., Carter, A., Messinger, D., Yirmiya, N., Zwaigenbaum, L., \& Stone, W. L. (2011). Recurrence risk for autism spectrum disorders: a baby siblings research consortium study. Pediatrics, 128(3), e1-e8. DOI: http://doi.org/10.1542/ peds.2010-2825.

Paula, C. S., Ribeiro, S. H. B., \& Teixeira, M. C. T. V. (2011). Epidemiologia e transtornos globais do desenvolvimento (pp. 151-158). In J. S. Schwartzman \& C. A. Araújo. (Ed.). Transtornos do espectro do autismo. São Paulo: Memnon Edições Científicas.

Paula, C. S., Zaqueu, L. da C. C., Vicente, T., Lowenhal, R., \& Miranda, C. (2011). Atuação de psicólogos e estruturação de serviços públicos na assistência à saúde mental de crianças e adolescentes. Psicologia: Teoria e Prática, 13(3), 81-95.

Paula, C. S., Lauridsen-Ribeiro, E., Wissow, L., Bordin, I. A. S., \& Evans-Lacko, S. (2012). How to improve the mental health care of children and adolescents in Brazil: Actions needed in the public sector. Revista Brasileira de Psiquiatria, 34(3), 334-341.

Reichow, B. (2012). Overview of meta-analyses on early intensive behavioral intervention for young children with autism spectrum disorders. Journal of Autism and Developmental Disorders, 42(4), 512-520.

Ronchi, J. P., \& Avellar, L. Z. (2010). Saúde mental da criança e do adolescente: a experiência do Capsi da cidade de Vitória-ES. Psicologia: Teoria e Prática, 12(1), 71-84.

Samms-Vaughan, M. E. (2014). The status of early identification and early intervention in autism spectrum disorders in lower- and middle-income countries. International Journal of Speech-Language Pathology, 16(1), 30-35.

Soltau, B., Biedermann, J., Hennicke, K., \& Fydrich, T. (2015). Mental health needs and availability of mental health care for children and adolescents with intellectual disability in Berlin. Journal of Intellectual Disability Research, 59(11), 983-994. 
Trembath, D., \& Vivanti, G. (2014). Problematic but predictive: individual differences in children with autism spectrum disorders. International Journal of Speech-Language Pathology, 16(1), 57-60.

Virués-Ortega, J. (2010). Applied behavior analytic intervention for autism in early childhood: meta-analysis, meta-regression and dose-response meta-analysis of multiple outcomes. Clinical Psychology Review, 30(4), 387-399.

Werner, E., Dawson, G., Munson, J., \& Osterling, J. (2005). Variation in Early Developmental Course in Autism and its Relation with Behavioral Outcome at 3-4 Years of Age. Journal of Autism and Developmental Disorders, 35(3), 337-350.

Zanon, R. B., Backes, B., \& Bosa, C. A. (2014). Identificação dos primeiros sintomas do autismo pelos pais. Psicologia: Teoria e Pesquisa, 30(1), 25-33.

Submissão: 2.6.2015

Aceitação: 19.11.2015 